\title{
Advances in Diagnostic Approaches of SARS-Cov-2 Virus
}

\author{
Ramakrishnan $V^{*}$, Vaishak $K$ and Barath $R$ \\ Human Cytogenetics laboratory, Faculty of Allied Health Sciences, Chettinad Academy of Research and \\ Education, India
}

\begin{abstract}
COVID-19 is caused by the SARS-CoV-2 virus, first identified as viral pneumonia, and later became a worldwide pandemic situation in December 2019. At present, there are 3,29,68,853 confirmed cases all over the world and 9,95,836 deaths $\left(27^{\text {th }}\right.$ September 2020; $\left.1.08 \mathrm{pm}\right)$, USA is first on the list of confirmed and death cases. It can cause ARDS which is the worst-case scenario where the oxygen level is dangerously less and patients are kept under ventilation. 'Prevention is better than cure', there is no effective vaccine productions and specific therapeutics options are not standard in many countries. Personal hygiene and sanitization can help prevent this nCoV. Diagnostic methods can help detect the virus so that recommended measures can be taken to safeguard the patient's life. Scientists are researching for future therapeutic options and vaccine production, also many clinical trials are going on for detecting the drug's efficacy in viral load is reduced in SARS-CoV-2 patients.
\end{abstract}

Keywords

SARS-CoV-2, Prevention, Diagnostic approaches

\begin{abstract}
Abbreviations
COVID-19: Corona Virus Disease 2019; ARDS: Acute Respiratory Distress Syndrome; QRT-PCR: Quantitative Real TimePolymerase Chain Reaction; CT: Computed Tomography; LUS: Lung Ultrasound; Al: Artificial intelligence
\end{abstract}

\section{Introduction}

The COVID-19 was first likely to have originated in the Huanan seafood market in Wuhan city Hubei province, Central China, even though the initial source of origination is not known still. It was first recorded as viral pneumonia and later it became a world-wide pandemic situation. Corona viruses fall under the Corona viridae family and Ortho Corona viridae subfamily [1]. It causes infections like a common cold, respiratory problems, and more severe problems like SARS and MERS and its main source of spread is the respiratory tract. Elderly people are likely to be more associated with ARDS and cytokine storms [2]. This virus has the potential to grow and become a world-wide pandemic, on $28^{\text {th }}$ of February 2020 a very high-level threat to the CoV epidemic has been raised by the WHO. SARS-CoV-2 transmission is faster and higher compared to previous viruses [3]. Through small droplets from the nose or mouth, it can easily spread from person-person through sneezing, coughing and speaking. Among 1 out of every 5 people is affected by a corona virus and becomes severely sick [4].

\section{Prevalence}

The outburst of COVID-19 pandemic originated from Huanan seafood market, china. As per WHO, the samples taken from the surroundings of this market were marked positive for this infection. Yet the interrelations among the infections with distinct animals have not been identified but the previ- ous record says that bats are the host for more than 30 infections which concludes that it might be a source for COVID-19 $[5,6]$. On $11^{\text {th }}$ of March, 2020 considering the uplift in severity and unfavorable consequences of the infection, WHO proclaimed it as an overall pandemic [7]. It has been retrieved from the WHO; up to $27^{\text {th }}$ of September 2020 the complete count of positive cases registered worldwide was 3,29,68,853 and the worldwide death rate rose up to $9,95,836$. As per the evidence provided by WHO, as of 27 September 2020 USA stands first in position worldwide and contributes to about $21.25 \%$ of total positive cases followed by India (18.42\%), Brazil (14.31\%), Russia (3.49\%) \& Columbia (2.44\%). Countries like Maldives (0.03\%), Myanmar $(0.028 \%)$ Zimbabwe $(0.024 \%)$, Thailand $(0.0108 \%)$ are least affected and stand down in position is illustrated in the below (Figure 1 and Figure 2).

*Corresponding author: Ramakrishnan V, Faculty of Allied Health Sciences, Chettinad Academy of Research and Education, Chettinad Health City, Kelambakkam - 603 103, Tamil Nadu, India

Accepted: November 11, 2020

Published online: November 13, 2020

Citation: Ramakrishnan V, Vaishak K, Barath R (2020) Advances in Diagnostic Approaches of SARS-Cov-2 Virus. Annals Gynecol Obstet 4(2):85-89 


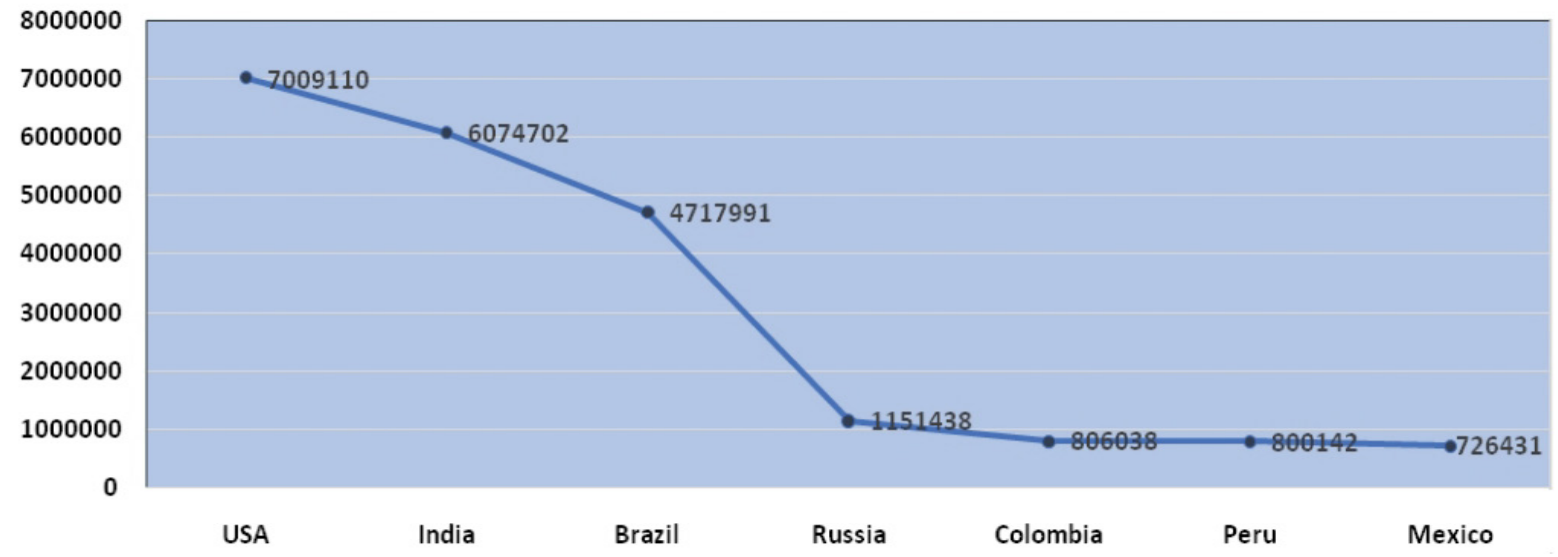

Figure 1: Number of positive cases according to each country till $27^{\text {th }}$ September 2020.

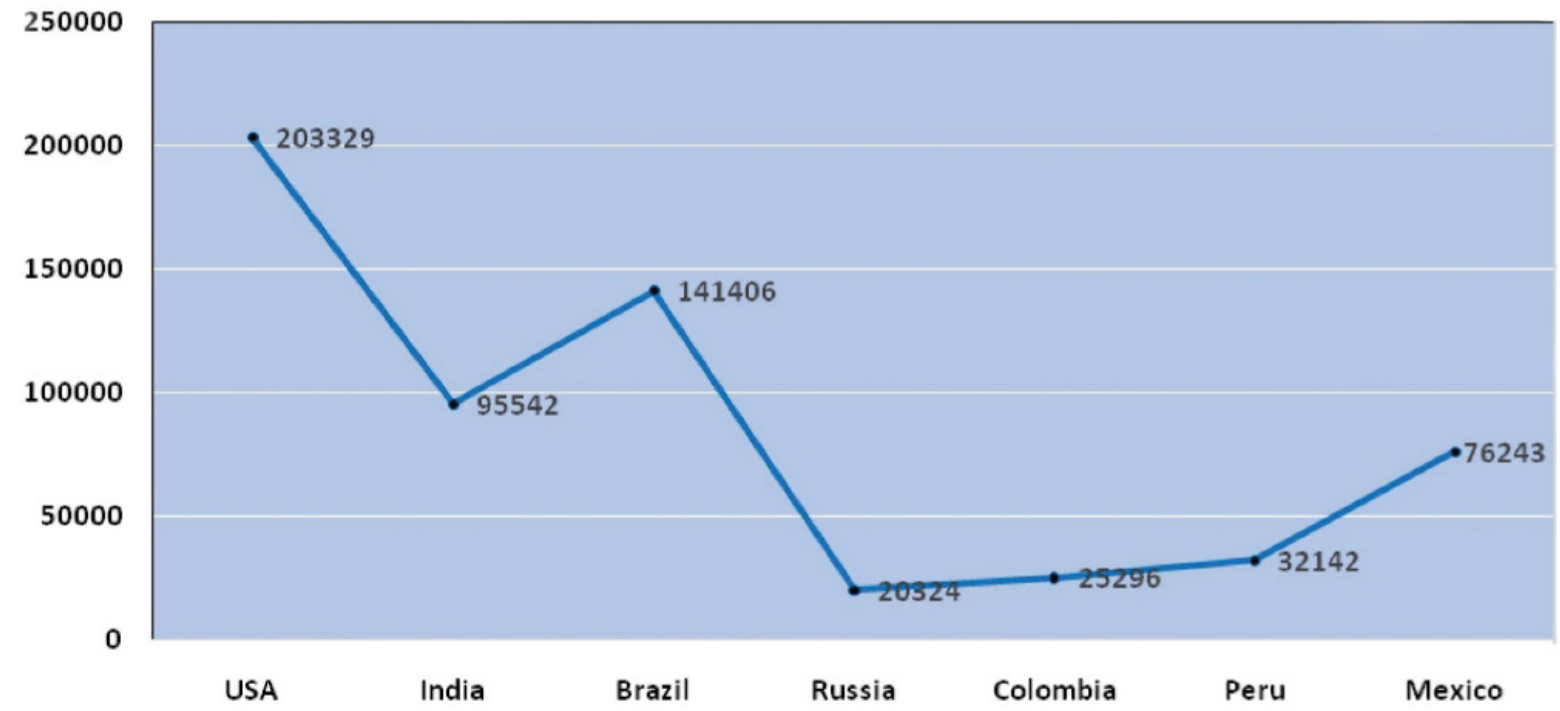

Figure 2: Number of death cases according to each country till $27^{\text {th }}$ September 2020.

\section{Mechanism}

The ingress of SARS-COV-2 into the cell which is being encountered commences with the synergy of Spike proteins (S - Proteins) made up of Glycoprotein with the receptors of the host. As per the types of viruses, the sites of Receptor Binding Domains (RBD) within the S1 locale of a COVID S protein differ. Some may have RBD at N-terminus for e.g. MHV and some may have such at C-terminus as in the case of SARS-COV $[8,9]$. At that point, viral particles access over host cell cytosol which can generally be achieved by Cathepsin or other proteases like TMPRSS2 etc. This helps in cleaving S-protein, which usually gets cleaved at 2 different sites of which the first cleavage is vital for division of Receptor Binding Domain \& fusion domains of $S$ protein. Subsequently, the second cleavage for revealing the fusion peptides [10,11], after the cell entry, the RNA gets uncoated and the replicase gene on the RNA strand is translated into two replicase poly proteins, further the proteinases of virus process these poly proteins to obtain independent proteins of replicase enzymes. These proteins bring about the output of full-length negative sense RNA and it functions as positive sense RNA template in future and the full-length negative sense RNA is reproduced to deliver shorter mRNAs [12].

\section{Diagnostic Approaches - Molecular Aspect \\ Quantitative real-time reverse transcription- Polymerase chain reaction (QRT-PCR)}

One of the most common and promising methods of diagnosis used in the detection COVID-19 is Quantitative Real-Time Reverse Transcriptase-Polymerase Chain Reaction [13]. The steps involved in this diagnostic method are as follows: 1) Extraction of RNA samples from the patients; 2) The RNA extracted is converted into cDNA by reverse transcription and it is amplified by specific fragments of cDNA by PCR; 3) Fluorescent signals are studied. When the Cycle threshold (C) values are lesser, it is an indication of diagnostic results as positive. Generally, it takes about 2-4 hour's when performed under trained people in a clinical laboratory whereas; automated QRT-PCR is expected to give the result in less than an hour. This method is very useful for diagnosis in early stages compared to antigen-antibody reaction [14]. If there is any 
other material that is involved it will affect the results and will show as 'false-positive results. Moreover, micro fluidic digital array PCR helps to lower the false-positive rate because it gives gene qualification at single molecule level [15].

\section{Detection by CRISPR/CAS Technology}

CRISPR/Cas system is a kind of impressive genetic engineering tool that mainly focuses on editing the desired genome of interest $[16,17]$. These technologies are now freshly introduced in detecting the viral particles with more promising results $[18,19]$. Major components of this technology include the CAS proteins which give the enzymatic apparatus needed to the procurement of new spacers from, targeting, attacking components, the guide RNAs helps in target sequence selectivity for the system and the fragments of target DNA $[20,21]$. The Cas proteins like $12 a \& 13$ a results in the guaranteed cleavage when bound over the target, in which a nonspecific single stranded DNA or RNA marked with fluorescent dye gets digested and released out $[22,23]$. Recent studies say that these CRISPR/Cas 13a tests alongside with isothermal amplification can locate SARS-COV-2 which was affirmed already by the US FDA for crisis use [24,25]. Also, the combination of cas12a with RT- LAMP shows a greater relevance and sensitivity over the samples [26]. Therefore, this technology is assessed as a promising diagnostic method for the detection of SARS-COV-2, since it can be incorporated with any nucleic acid augmentation frameworks $[27,28]$.

\section{Detection by genome sequencing}

Genome sequencing is a kind of methodology in which the arrangement of DNA nucleotides or the bases in the whole genome and the composition of double helix DNA are unraveled [29]. It is notable for its agility and precision in recognizing the unknown viral particles entering our body and also it has a numerous point of interest in virus detection. To start with, it empowers examination of the SARS-CoV-2 develop- ment during transmission and following of COVID developments [30]. It is then followed by viable prohibition of other microbes. Also, genomic sequencing can distinguish numerous microorganisms existing in a solitary patient, which helps execute sensible medications but requires lavish equipment and skilled personnel [31]. Higher recognition efficiency can be achieved by using droplet based single cell sequencing technology. At present, this method has first concern at the ongoing phase of the epidemic, yet patient's genome sequencing will provide a better understanding about the human susceptibility over the SARS-COV-2 infection [32]. By interpreting the genomic sequences of the affected patients, the transmission dynamics of SARS-COV-2 can be very well understood which aids in the rapid development of vaccines against this infection and their diagnostic approaches is illustrated in (Figure 3).

\section{Imaging Examination}

\section{Chest computed tomography (CT)}

It is an $\mathrm{x}$-ray imaging technique where narrow $\mathrm{x}$-ray beams are focused on patients' body; it gives the cross-sectional images of the required target [33]. In this aspect, it is focused on cross-sectional images of the lungs by CT where we get more details about radiographic features in most of the COVID-19 patients [34]. CT scans are strongly recommended to use as a diagnostic option, there are five stages classified based on CT imaging stages $[35,36]$ is presented in (Table 1 ).

\section{Lung ultrasound (LUS)}

Ultrasound is used to image the internals of the body; it is done by the production of sound waves by probes or transducers above human hearing threshold frequencies, these probes can also be sent inside the body to get more detailed image [37]. LUS is an easy option for diagnosis of suspected COVID-19 patients because, it can be done at any stage of diagnostics or therapeutic processes while the patients still

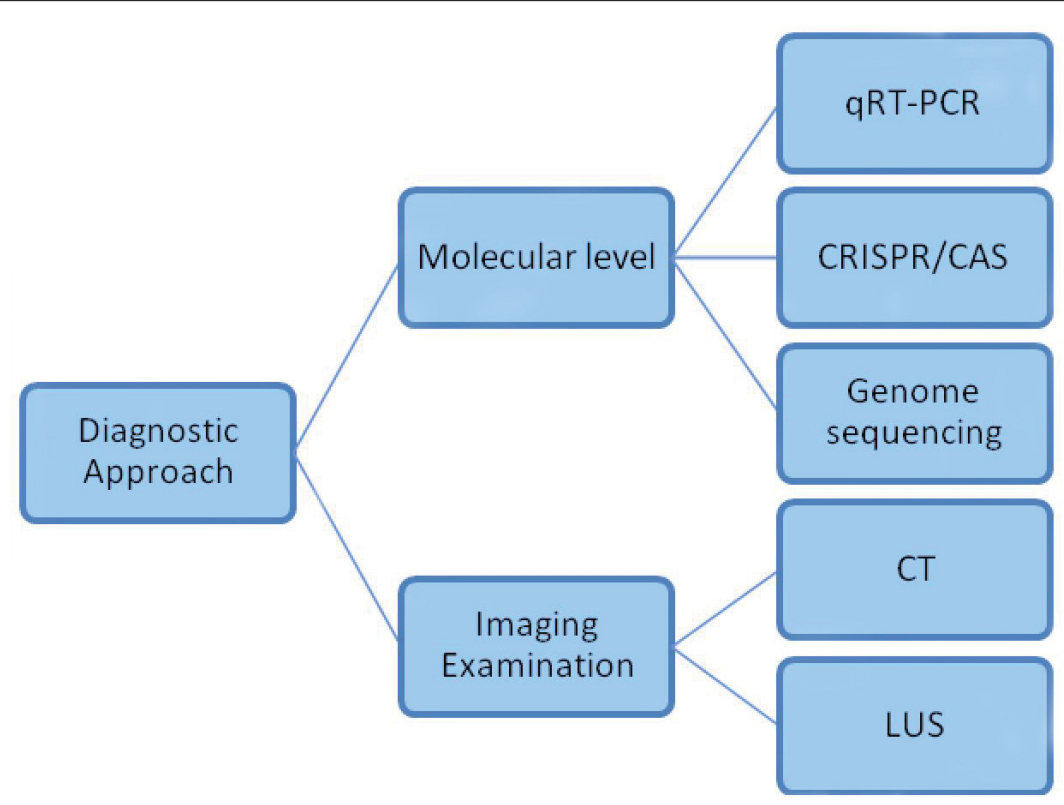

Figure 3: The diagnostic approaches in SARS-CoV-2 virus. 
Table 1: Stages based on CT image in SARS-CoV-2 virus.

\begin{tabular}{|c|l|l|}
\hline S.No & Stages based on CT image & \multicolumn{1}{|c|}{ Conditions according to each stage } \\
\hline 1 & Ultra-early stage & $\begin{array}{l}\text { No clinical manifestation in this stage, laboratory results are negative but tests positive for throat } \\
\text { swab generally between 1-2 weeks after exposure to contaminated areas. }\end{array}$ \\
\hline 2 & Early stage & $\begin{array}{l}\text { Initial period within three days once encountered with cold, wheeze, dry cough, pyrexia etc., it } \\
\text { shows particular numerous strewed irregulars or groups of ground-glass opacities, and divided by } \\
\text { beehive-like stiffen interlobular septa. }\end{array}$ \\
\hline 3 & Rapid Progression stage & $\begin{array}{l}\text { Period of three to seven days after being influenced by the virus, a merged \&huge-scale light } \\
\text { coalesce with air-bronchogram inside was manifested by CT. }\end{array}$ \\
\hline 4 & Consolidation stage & $\begin{array}{l}\text { Period of about one to two weeks after exposure to clinical manifestation, CT image portrayed } \\
\text { several strewed coalesce in lower density and lesser range than the before stage. }\end{array}$ \\
\hline 5 & Dissipation stage & $\begin{array}{l}\text { Hardly between 2-3 weeks after clinical manifestation, furthermore was the range of lesions } \\
\text { reduced. Patchy consolidation or strip-like opacity was shown by CT image, after sometime grid- } \\
\text { like denser interlobular septum, thickening and strip-like twist of bronchial wall and less scattered } \\
\text { patchy consolidations was observed. }\end{array}$ \\
\hline
\end{tabular}

on bedside [38]. There are some advantages compared to CT technique, LUS has no radiation, it is low cost and can be performed repeatedly faster $[39,40]$. LUS has limitations when compared to $\mathrm{CT}$; low sensitivity, lesions that are presented deep and intrapulmonary cannot be detected [41].

\section{Treatment}

There is no particular therapeutic option available for COVID-19 and vaccines are not yet discovered, scientists are undergoing research for developing vaccines and having treatment plans for future aspects [42], vaccine as an early preventive strategy for SARS-CoV-2 virus. There are many antiviral drugs like hydroxycloroquine and Lopinavir/Ritonavir were taken by people for treatment [43]. There are some selective treatment options based on the $7^{\text {th }}$ edition of 'COVID-19 diagnosis and treatment guideline' released by $\mathrm{NCH}$ of the people's Republic of China, it is based on three categories are as follows: 1 . Standard treatment; 2 . Treatment for severe and critical cases; 3 . Traditional Chinese medicine [44].

\section{Conclusion}

SARS-CoV-2 is a highly infectious and transferrable virus which causes severe conditions, even though there are no therapeutic options and vaccines available. Diagnostics methods (Molecular aspect and Imaging examination) are there to detect the virus but, improvements and advancements are much needed to faster the rate of diagnosis. The use of $\mathrm{Al}$ technology can also be helpful in diagnostic aspects like; robots can be programmed for collecting samples which reduces human force. In near future, the advanced treatment options of vaccines production and molecular diagnostic methods will provide a great help for disease diagnosis and detection.

\section{Acknowledgement}

Authors thank Chettinad Academy of Research and Education (CARE) for support.

\section{References}

1. Heng Li, Shang-Ming Liu, Xiao-Hua Yu, et al. (2020) Corona virus disease 2019 (COVID-19): Current status and future perspectives. Int J Antimicrob Agents 55: 105951.
2. Yan-Rong Guo, Qing-Dong Cao, Zhong-Si Hong, et al. (2020) The origin, transmission and clinical therapies on corona virus disease 2019 (COVID-19) outbreak - an update on the status. Mil Med Res 7: 11.

3. David S C Hui, Alimuddin Zumla (2019) Severe acute respiratory syndrome: Historical, epidemiologic, and clinical features. Infect Dis Clin North Am 33: 869-889.

4. (2020) World Health Organization. WHO.

5. Lisa E Gralinski, Vineet D Menachery (2020) Return of the corona virus: 2019-nCoV. Viruses 12: 135.

6. Tung Phan (2020) Novel corona virus: From discovery to clinical diagnostics. Infect Genet Evol 79: 104211.

7. Jaffar A, Al-Tawfiq, Roberto Leonardi, et al. (2020) Prevalence and fatality rates of COVID-19: What are the reasons for the wide variations worldwide? Travel Med Infect Dis 35: 101711.

8. Cheng P K, Wong D A, Tong LK, et al. (2004) Viral shedding patterns of corona virus in patients with probable severe acute respiratory syndrome. Lancet 363: 1699-700.

9. Kubo H, Yamada Y K, Taguchi F (1994) Localization of neutralizing epitopes and the receptor-binding site within the amino-terminal 330 amino acids of the murine corona virus spike protein. $J$ Virol 68: 5403-5410.

10. Sandrine Belouzard, Victor C Chu, Gary R Whittaker (2009) ACtivation of the SARS corona virus spike protein via sequential proteolytic cleavage at two distinct sites. Proc Natl Acad Sci U S A 106: 5871-5876.

11. Bosch B J, van der Zee R, de Haan C A, et al. (2003) The corona virus spike protein is a class I virus fusion protein: Structural and functional characterization of the fusion core complex. J Viral 77: 8801-8811.

12. Zhiqi Song, Yanfeng Xu, Linlin Bao, et al. (2019) From SARS to MERS, Thrusting corona viruses into the spotlight. Viruses 11: 59.

13. Yishan Wang, Hanyujie Kang, Xuefeng Liu, et al. (2020) Combination of RT-QPCR testing and clinical features for diagnosis of COVID-19 facilitates management of SARS-CoV-2 outbreak. J Med Virol 92: 538-539.

14. Xi Yuan, Chengming Yang, Qian He, et al. (2020) Current and perspective diagnostic techniques for COVID-19. ACS Infect Dis 6: 1998-2016.

15. Shufang Zhou, Tong Gou, Jiumei Hu, et al. (2019) A highly integrated real-time digital PCR device for accurate DNA quantitative analysis. Biosens Bioelectron 128: 151-158. 
16. Anzalone AV, Randolph PB, Davis JR, et al. (2019) Search-andReplace genome editing without double-strand breaks or donor DNA. Nature 576: 149-157.

17. Qin P, Parlak M, Kuscu C, et al. (2017) Live cell imaging of lowand non-repetitive chromosome loci using CRISPR-Cas9. Nat Commun 8: 14725 .

18. Flint $M$, Chatterjee P, Lin DL, et al. (2019) A genome-wide CRISPR screen identifies $\mathrm{N}$-acetylglucosamine-1-phosphate transferase as a potential antiviral target for Ebola virus. Nat Commun 10: 285.

19. He Q, Yu D, Bao M, et al. (2020) High-throughput and all-solution phase African swine fever virus (ASFV) detection using crispr-cas12aandfluorescence based point-of-care system. Biosens Bioelectron 154: 112068.

20. Corinna Richter, James T Chang, Peter C Fineran (2012) Function and regulation of clustered regularly interspaced short palindromic repeats (CRISPR)/CRISPR associated (CAS) systems. Viruses 4: 2291-2311.

21. Guanqing Liu, Yong Zhang, Tao Zhang (2019) Computational approaches for effective CRISPR guide RNA design and evaluation. Comput Struct Biotechnol J 18: 35-44.

22. Jin Zhou, Lijuan Yin, Yanan Dong, et al. (2020) CRISPR-Cas13a based bacterial detection platform: sensing pathogen staphylococcus aureus in food samples. Analytica Chimica Acta 1127: 225-233.

23. Peiwu Qin, Myeongkee Park, Kendra J Alfson, et al. (2019) Rapid and fully micro fluidic ebola virus detection with CRISPR-Cas13a. ACS Sens 4: 1048-1054.

24. Zhang F, Abudayyeh OO, Jonathan SG (2020) A protocol for detection of COVID-19 using CRISPR diagnostics.

25. Hou T, Zeng W, Yang M, et al. (2020) Development and evaluation of A CRISPR-based diagnostic for 2019-novel corona virus. MedRxiv 10: 1101.

26. Broughton JP, Deng X, Yu G, et al. (2020) CRISPR-Cas12-based detection of SARS-CoV-2. Nat Biotechnol 38: 870-874.

27. Kellner MJ, Koob JG, Gootenberg JS, et al. (2019) Sherlock: Nucleic acid detection with CRISPR nucleases. Nat Protoc 14: 2986-3012.

28. Xinjie Wang, Xiaoyun Shang, Xingxu Huang (2020) Next-generation pathogen diagnosis with CRISPR/Cas-based detection methods. Emerg Microbes Infect 9: 1682-1691.

29. Kathryn A Phillips, Julia Trosman, Robin K Kelley, et al. (2014) Genomic sequencing: Assessing the health care system, policy, and big-data implications. Health Aff (Millwood) 33: 1246-1253.
30. Kupferschmidt K (2020) Genome analyses help track corona virus' moves. Science 367: 1176-1177.

31. Wang Y, Cao T, Ko J, et al. (2020) Dissolvable polyacrylamide beads for high-throughput droplet DNA bar coding. Adv Sci 7: 1903463.

32. Chen $Y$, Liu Q, Guo D (2020) Emerging corona viruses: Genome structure, replication, and pathogenesis. J Med Virol 92: 418-423.

33. https://www.nibib.nih.gov/science-education/science-topics/ computed-tomography-ct

34. Michael Chung, Adam Bernheim, Xueyan Mei, et al. (2020) CT imaging features of 2019 novel corona virus (2019-nCoV). Radiology 295: 202-207.

35. Yuliang Liu 1, Guohua Liu, Quan Zhang (2020) Deep learning and medical diagnosis. The Lancet 394: 1709-1710.

36. Ying-Hui Jin, Lin Cai, Zhen-Shun Cheng, et al. (2020) A rapid advice guideline for the diagnosis and treatment of 2019 novel corona virus (2019-nCoV) infected pneumonia (standard version). Mil Med Res 7: 4.

37. https://www.nibib.nih.gov/science-education/science-topics/ ultrasound

38. Natalia Buda, Elena Segura-Grau, Jolanta Cylwik, et al. (2020) Lung ultrasound in the diagnosis of COVID-19 infection - A case series and review of the literature. Adv Med Sci 65: 378-385.

39. Qian-Yi Peng, Xiao-Ting Wang, Li-Na Zhang (2020) Chinese critical care ultrasound study group (CCUSG) findings of lung ultrasonography of novel corona virus pneumonia during the 20192020 epidemic. Intensive Care Med 46: 849-850.

40. D Buonsenso, A Piano, F Raffaelli, et al. (2020) Point-of-care lung ultrasound findings in novel corona virus disease-19 pneumoniae: A case report and potential applications during COVID-19 outbreak. Eur Rev Med Pharmacol Sci 24: 2776-2780.

41. Neda khalili, Sara Haseli, Pooya Iranpour (2020) Lung ultrasound in covid-19 pneumonia: Prospects and limitations. Acad Radiol 27: 1044-1045.

42. Marco Cascella, Michael Rajnik, Arturo Cuomo, et al. (2020) Features, evaluation, and treatment of corona virus (COVID-19) Stat Pearls.

43. Melika Lotfi, Michael R Hamblin, Nima Rezaeif (2020) COVID-19: Transmission, prevention, and potential therapeutic opportunities. Clin Chim Acta 508: 254-266.

44. Yang Song, Min Zhang, Ling Yin, et al. (2020) COVID-19 treatment: Close to a cure? A rapid review of pharmacotherapies for the novel corona virus (SARS-CoV-2). Int J Antimicrob Agents 56: 106080.

DOI: $10.36959 / 468 / 472$

Copyright: (C) 2020 Ramakrishnan V, et al. This is an open-access article distributed under the terms of the Creative Commons Attribution License, which permits unrestricted use, distribution, and reproduction in any medium, provided the original author and source are credited. 